\title{
Criando contextos ecológicos de desenvolvimento e direitos humanos para adolescentes $^{1}$
}

\author{
Ana Paula Lazzaretti de Souza ${ }^{2}$ \\ Luciana Dutra-Thomé \\ Eva Diniz Bensaja Dei Schiró \\ Camila de Aquino Morais \\ Silvia Helena Koller \\ Universidade Federal do Rio Grande do Sul, Porto Alegre-RS, Brasil
}

\begin{abstract}
Resumo: Neste estudo descreve-se a aplicação do método de Inserção Ecológica em um programa de intervenção para educação em direitos humanos e protagonismo juvenil para adolescentes em situação de vulnerabilidade social. A criação de um microssistema constituído pelos participantes e pela equipe executora do programa resulta em mudanças positivas de comportamento e respeito às regras, entre outros. Estas transformações surgem em consonância com a proposta da Abordagem Bioecológica do Desenvolvimento Humano, sobre a influência do ambiente. Este estudo reforça a importância da aplicação dos resultados em políticas públicas e sociais.
\end{abstract}

Palavras-chave: métodos, desenvolvimento do adolescente, educação, direitos humanos.

\section{Creating ecological contexts of development and human rights for adolescents}

\begin{abstract}
This paper describes the application of the Ecological Engagement method in an intervention program for human rights education and agency, targeting adolescents in situations of social vulnerability. A new microsystem was built and was possible to identify social changes in the participants' behavior, respect for the rules, etc. These changes were consistent with the proposed approach of the Bioecological model of human development on the influence of environment. These findings reinforced the importance of applying the results in public and social polices.
\end{abstract}

Keywords: methods, adolescent development, education, human rights.

\section{La creación de contextos ecológicos del desarrollo y derechos humanos para los adolescentes}

\begin{abstract}
Resumen: Este artículo describe la aplicación del método de Inserción Ecológica en un programa de intervención para educación en derechos humanos y protagonismo juvenil, dirigido a adolescentes en situaciones de vulnerabilidad social. La creación de un nuevo microsistema resulta en cambios positivos en el comportamiento, respecto por las reglas, entre otros. Estos cambios surgen en consonancia con la propuesta del enfoque bioecológico para el desarrollo humano y la influencia del ambiente. La realización de este estudio refuerza la importancia de aplicar los resultados en las políticas públicas y sociales.
\end{abstract}

Palabras clave: métodos, desarrollo del adolescente, educación, derechos humanos.

A Inserção Ecológica é um método de investigação no qual os pesquisadores se inserem no contexto natural dos participantes (Cecconello \& Koller, 2003). Contextos naturais influenciam no desenvolvimento dos indivíduos, pela estabilidade nas relações, respeito à hierarquia de poder e presença de laços afetivos e reciprocidade. Este relato de uma experiência de pesquisa-intervenção baseia-se em programa de educação em direitos humanos e protagonismo para adolescentes em situação de vulnerabilidade social, realizado com o método de inserção ecológica.

\footnotetext{
1 Este texto foi revisado seguindo o Acordo Ortográfico da Língua Portuguesa (1990), em vigor a partir de $1^{\circ}$ de janeiro de 2009 . O programa de intervenção Juventude em Cena foi financiado pelo Ministério da Educação (MEC - Editais Proext 2007 e 2008).

${ }^{2}$ Endereço para correspondência:

Ana Paula Lazzaretti de Souza. Rua Ramiro Barcelos, 2600, sala 104. CEP 90.035-003. Porto Alegre-RS, Brasil. E-mail:

anapaula.lazzaretti@gmail.com
}

A Abordagem Bioecológica do Desenvolvimento Humano $(\mathrm{ABDH})$ propõe-se a compreender os fenômenos do desenvolvimento, ao longo do ciclo vital, em uma perspectiva histórica, cultural e interacionista. O ambiente ecológico foi descrito por Bronfenbrenner (1979/1996), como um conjunto de estruturas concêntricas no qual a pessoa se insere. É uma sequência de eventos que envolve a transformação biológica do organismo pelo ciclo vital. A relação da pessoa com o ambiente é multidirecional e concomitante, ambos se influenciam e desenvolvem (Bronfenbrenner \& Evans, 2000).

O modelo de desenvolvimento, com base nas dimensões processo, pessoa, contexto e tempo (PPCT), foi proposto como produto do intercruzamento destas (Bronfenbrenner, 1979/1996). A percepção do desenvolvimento-no-contexto (Cecconello \& Koller, 2003) inclui vários níveis de análise da influência do ambiente no processo de desenvolvimento, que serão descritos a seguir.

(1) Pessoa: as características biológicas, físicas e psicológicas em interação com o ambiente são tanto produto quanto 
produtoras do desenvolvimento e influenciam os processos proximais por sua demanda, recurso e força (Bronfenbrenner, 1993, 1995; Bronfenbrenner \& Morris, 1998).

(2) Tempo: cada processo desenvolvimental particular e único é influenciado pelo tempo, pelas políticas e pelos valores dominantes. Indivíduos e grupos da mesma geração partilham histórias de vida e experiências (Bronfenbrenner, 1988). O desenvolvimento é compreendido como um processo marcado pela constância e transformação (Bronfenbrenner, 2005).

(3) Contexto: o microssistema ou ambiente imediato da pessoa - trabalho, casa, creche - propicia interações face a face, com estabilidade e reciprocidade, para promover os processos proximais. Mesossistema é a inter-relação de microssistemas. Exossistema é um sistema no qual a pessoa não participa, mas recebe sua influência. Macrossistema reúne aspectos sociais, culturais, crenças, valores e ideologias dos demais (Bronfenbrenner, 1979/1996).

(4) Processo: o padrão de interação da pessoa com o ambiente que engloba outras pessoas, objetos e símbolos é definido como processo proximal, ou seja, é o motor do desenvolvimento (Bronfenbrenner, 1995; Bronfenbrenner \& Morris, 1998). Estes são fundamentais para a criação de novos microssistemas.

(5) Inserção ecológica: o desenvolvimento acontece pelo estabelecimento de processos proximais em um contexto de uma pessoa, envolvendo seu tempo histórico e processos proximais (Cecconello \& Koller, 2003).

Neste relato de experiência, foi dada uma nova perspectiva acerca da inserção ecológica pela criação de um novo microssistema, inexistente na história anterior dos participantes, mas que permitiu o estabelecimento de relações estáveis, recíprocas e face a face. Pesquisadores/ equipe de intervenção e participantes em processo de desenvolvimento aplicaram um programa de educação em direitos humanos. Anteriormente, inserir-se ecologicamente consistia na ação de deslocar-se para o contexto no qual se propunha o estudo (Aquino-Morais, 2008; Cecconello, 2003; Morais, 2009; Vasconcelos, Yunes, \& Garcia, 2009). Tanto participantes quanto pesquisadores vivenciaram um espaço de transição ecológica para novos papéis, pois, ao mesmo tempo em que estavam inseridos ecologicamente no microssistema, o construíam.

Os processos proximais foram centrais para participação em atividades comuns mantidas com base regular de tempo, progressivamente mais complexas, despertando atenção e interesse de todos, com reciprocidade e disponibilidade e foco comum na educação para os direitos humanos. As supervisões regulares permitiram a discussão e a busca de soluções, avaliação do trabalho e estudo de casos individualizados. Foi espaço ecológico de compartilhamento dos sentimentos e percepções vividos pela equipe e participantes. O objetivo deste relato é, portanto, apresentar uma nova maneira de utilizar o método da inserção ecológica, a partir da experiência no programa Juventude em Cena.

\section{Método}

\section{Programa de educação em direitos humanos}

O Juventude em Cena foi criado como um programa de ensino, pesquisa (aprovado pelo Comitê de Ética em Pesquisa do Instituto de Psicologia da UFRGS/Protocolo $n^{\text {o }}$ 2008/017) e extensão, com foco em preparar adolescentes para o enfrentamento das adversidades sociais dos seus contextos e estimular a participação ativa na vida política e social da sua comunidade. O conteúdo do programa de capacitação foi elaborado de acordo com os princípios da Convenção dos Direitos da Criança (ONU, 1989) e Estatuto da Criança e do Adolescente (ECA) (Lei No. 8.069, 1990) com base nos três "Ps" - provisão, proteção e participação. Esses princípios regulam o desenvolvimento da criança, a partir da provisão das suas necessidades, da proteção contra negligência e diversas formas de violência, além da participação nas decisões sobre suas vidas (Verhellen, 2000). Oito eixos transversais embasaram a intervenção: direitos da criança e do adolescente, identidade, solidariedade, cidadania, saúde, risco e proteção (violação de direitos, projetos de vida, incluindo escola, família e trabalho), políticas públicas, protagonismo juvenil/participação social. A teoria dos Quatro Pilares da Educação para o Desenvolvimento Humano também serviu de subsídio: "aprender a conhecer", "aprender a fazer", "aprender a conviver" e "aprender a ser" (Delors, 1998; Hassenpflug, 2004).

\section{Participantes}

O programa iniciou, em 2008, com 70 adolescentes, de ambos os sexos, com idades entre 12 e 18 anos. Os participantes eram integrantes de programas sociais e compareciam à intervenção acompanhados por técnicos da rede de proteção infanto-juvenil de seus municípios de origem, que fazem parte da Região Metropolitana de Porto Alegre-RS.

\section{Procedimento}

Foram realizados 10 encontros quinzenais ao longo de cinco meses. Os encontros consistiram em instrumentalização teórica, com uso de filmes e exposição das temáticas como cidadania, protagonismo, direitos tanto para os técnicos quanto para os adolescentes. Em seguida, os adolescentes participavam de dinâmicas em pequenos grupos. O fechamento dos conteúdos trabalhados baseava-se em reflexão sobre as atividades, e todos tinham espaço para expressar o que haviam aprendido, visando à integração e conclusão objetiva da atividade. $\mathrm{O}$ trabalho com os técnicos possibilitou que se tornassem mediadores dos adolescentes no processo de protagonismo, eles proporcionaram a comunicação entre a equipe executora com os adolescentes no dia a dia na comunidade, constituindo-se como referência para o esclarecimento de dúvidas, orientações, suporte na execução das 
tarefas de casa. O processo da intervenção foi avaliado, no decorrer de sua execução, em reuniões de equipe semanais e no registro, em diário de campo, das impressões, sentimentos e observações por todos os membros da equipe.

\section{Resultados e Discussão}

\section{Inserção ecológica e a criação de um novo microssistema}

O formato do programa de intervenção configurou a emergência de um "novo" microssistema intitulado "Juventude em Cena" no qual se desenvolveram as atividades que possibilitaram o estabelecimento de processos proximais entre os participantes e a equipe de execução. Tal constituição foi marcada pelas novas interações com pessoas, objetos e símbolos, em um ambiente diferente daqueles que os adolescentes costumavam ter acesso. A intervenção aconteceu nas instalações da universidade, o que implicava a inserção dos participantes em novo ambiente, contato com recursos desse local que representa a escolarização, o saber e o conhecimento científico. Ademais, a configuração do espaço físico ofereceu novas experiências que, embora simples, eram inusitadas no cotidiano desses adolescentes. Na equipe, esteve sempre presente o cuidado com expectativas e necessidades do grupo, na tentativa de criar uma relação de reciprocidade entre todos. A adesão crescente às atividades mostrou a adaptação das expectativas de todos às propostas da intervenção.

Impressões diversas foram geradas, a partir do contato com os participantes. Em um primeiro momento, a equipe se deparou com adolescentes agitados, com necessidade de supervisão, sempre curiosos, mas às vezes desconfiados e inicialmente resistentes à intervenção. As interações de adolescentes e técnicos traduziram a influência - a partir da manifestação das características de demanda da pessoa no ambiente e traziam informações do seu contexto para o novo microssistema que estava se formando. Nesse sentido, o contato gradualmente mais próximo com os adolescentes permitiu que a equipe conhecesse de forma mais detalhada suas histórias de vida - características dos recursos da pessoa. Foram sempre necessárias diferentes maneiras de conduzir as atividades e de estabelecer relações - características diversas de força da pessoa - ao longo da intervenção. Os desafios desse tipo de trabalho e a necessidade de ajustes, durante sua realização, demonstravam a importância de estar atento não somente às peculiaridades dos contextos de origem dos participantes, mas das características de cada um e as da própria equipe.

Movimentos de superação significativos foram aparecendo ao longo do processo, uma vez que se tratava de adolescentes com oportunidades escassas e expostos a diversos fatores de risco - pobreza, violência e exploração. Tais atributos da pessoa não exerciam influência isolada no desenvolvimento, pois disposições, recursos e demandas influenciavam-se mutuamente e precisavam ser compreendidos, a partir da interação com diversos contextos que constituíam a rede social da pessoa (Bronfenbrenner \& Morris, 1998).

As atividades realizadas foram progressivamente mais complexas, pela integração de conteúdos - direitos, cidadania, protagonismo, entre outros -, propondo constante reflexão e crítica sobre a realidade vivida. À medida que os adolescentes entendiam as propostas e se sentiam à vontade com o grupo no microssistema criado, se expressavam a respeito dos temas e interagiam com os pares e com a equipe. Os pequenos grupos possibilitaram proximidade entre todos. Algumas tarefas permitiram o estabelecimento de interação progressivamente mais complexa, relacionada aos interesses e curiosidades com interação com objetos, símbolos e ambiente social e promovendo o seu desenvolvimento. A transformação de comportamentos, ao longo das tarefas, denotava-se nos processos de continuidade e mudança.

A equipe executora também percebeu mudanças em seus componentes, não apenas pessoais, como a revisão de valores e o dar-se conta das diferentes realidades da vida dos participantes do programa, mas também modificações nas relações entre seus membros. Portanto, os processos proximais no desenvolvimento conduziram à modificação de comportamentos em todo o grupo, que, ao longo das atividades e das relações estabelecidas, transformaram-se e se tornaram funcionais. Inicialmente, os adolescentes tinham uma atitude desordenada, barulhenta e pouco estruturada: as saídas das salas de atividades eram constantes, o espaço físico da faculdade foi danificado com grafites, o lixo era deixado pelo chão. Com o tempo, a modificação destas atitudes foi claramente percebida, com o respeito e adesão às regras propostas, assim como à preservação do espaço utilizado pelo grupo.

Os demais sistemas ecológicos (família, escola, comunidade) vivenciados pelos adolescentes puderam ser acessados. As interações contínuas, realizadas ao longo do processo de execução do programa, permitiram, por meio de conversas informais e de atividades estruturadas, conhecer e captar aspectos do contexto mais amplo. Os quatro níveis dos contextos de inter-relação dos participantes do programa (Bronfenbrenner, 1979/1996) foram mapeados. Assim, a realidade social, cultural e física do(s) contexto(s) dos adolescentes tornou-se presente de diferentes formas. Desse modo, mesmo não ocorrendo a inserção ecológica "tradicional" (Cecconello \& Koller, 2003), no próprio contexto de desenvolvimento cotidiano da pessoa, este foi apreendido de forma indireta, sem minimizar seu impacto e a importância no desenvolvimento.

No decorrer da intervenção, o microssistema proporcionou o estabelecimento de novas relações, o que caracterizou a transição ecológica a uma rede social ampliada (Bronfenbrenner, 1979/1996). Por exemplo, um adolescente, que havia iniciado o trabalho de aprendiz (Decreto №5598, 2005), compartilhou com o grupo sua decisão de economizar dinheiro para investir nos estudos. Na conversa estabelecida, ele contou que "sua mãe não acreditava que ele pudesse crescer na vida e ter melhores condições", afirmando que ele iria 
se "perder nas drogas como os outros meninos do bairro". Mas o adolescente afirmou querer mostrar à mãe que "seria capaz de fazer e ser diferente".

O macrossistema expressava-se pela realidade da pobreza e sua implicação social, o que perpassava as temáticas e as experiências relatadas. Ao longo dos encontros, uma das principais motivações dos adolescentes para estarem no programa era o fator alimentação. Para muitos esta provisão em suas casas era precária. Desse modo, a expectativa de ter almoço e lanche assumia um caráter motivador. Sabendo desta situação, a equipe buscou prover melhores refeições, a fim de suprir necessidades básicas dos adolescentes e, também, utilizar como estratégia de adesão ao longo do processo de vinculação com o programa e a equipe.

Outros fenômenos como a drogadição e a sexualidade estiveram presentes como aspectos que marcaram as influências macrossistêmicas. Durante o programa, a equipe identificou, por meio das conversas informais com os adolescentes, que estes estavam começando a ingerir bebidas alcoólicas. A equipe buscou por meio dos eixos temáticos já estabelecidos, como saúde, risco e proteção, criar espaços para abordar esses comportamentos. A via de compreensão foi norteada pelo reconhecimento da adolescência, suas tensões, bem como pelo contexto social e cultural.

Comentários sobre sexualidade/afetividade foram marcantes desde o início do programa, ao se iniciarem as "paqueras", entre eles, e as divergências. Criaram, então, um mural no qual pudessem trocar recados entre si, utilizavam os espaços de lazer e almoço para se aproximarem, verbalizavam em seus grupos de amigos acerca de um ou outro que lhes interessavam. A equipe reconheceu essa necessidade, propondo horários livres e espaços de convivência. Nas ocasiões em que algum preconceito ou homofobia foram aparentes, pontuaram-se o respeito e o reconhecimento do outro em sua individualidade.

O Juventude em Cena era constituído por adolescentes da mesma faixa etária, do mesmo nível socioeconômico e que partilharam, simultaneamente, um conjunto de atividades. $\mathrm{O}$ desenvolvimento deste programa ocorreu num tempo histórico, no qual se assistia, no contexto sociopolítico, à valorização dos direitos da criança e do adolescente, assim como, à importância do protagonismo juvenil. Além disso, despertou sentimentos e vivências, em função de outras experiências e acontecimentos passados, incorporados, transformados e atualizados no desenvolvimento vigente, influenciados pelo tempo histórico no qual ocorreram. Os acontecimentos exteriores ao macrotempo sociopolítico e cultural vigente influenciavam o seu desenvolvimento em momentos de vida e processos de maturação biológica equivalentes e foram incluídos no programa. Por exemplo, em época de eleições foi convidada uma figura política que era candidata, por sua trajetória de protagonismo e envolvimento político, desde a sua juventude, que conversou com o grupo sobre cidadania e intervenção política e social, como algo que poderia ser desenvolvido individualmente em suas vidas. O período de eleições configurava-se no macrotempo e influenciou o programa, pelas mudanças de administração política nos municípios. Tais mudanças exossistêmicas garantiram ou atrapalharam a participação e/ou permanência no programa.

A noção de microtempo implica o desenvolvimento de atividades numa base regular, seguida de atividades progressivamente mais complexas que mantêm a atenção dos envolvidos (Bronfenbrenner, 1977). Estes requisitos foram atendidos na medida em que essas atividades foram desenvolvidas ao longo de encontros quinzenais durante cinco meses, em um processo crescente de interação e conhecimento mútuo. O grau de complexidade das tarefas foi assegurado, ao se desenvolverem propostas novas, às quais os adolescentes e equipe, no seu dia a dia, não tinham possibilidade de aceder.

No decorrer do programa, a evasão de participantes; a não adesão de técnicos; e os desajustes no planejamento de atividades apareceram, mas eram esperados pelas dimensões e características do grupo. À medida que foram estabelecidos os processos proximais, foi possível perceber as necessidades de todos e adaptar as atividades e demais procedimentos para responder às expectativas. Atividades mais dinâmicas, como gincanas culturais, foram mudanças de estratégia para despertar maior interesse e engajamento às atividades. Na medida em que as dificuldades eram resolvidas e novas estratégias implantadas, foi possível notar o desenvolvimento dos processos proximais estabelecidos, adesão e cooperação com as atividades.

A centralidade do conceito de processo proximal na ABDH mostrou-se evidente, durante toda a inserção ecológica no programa, uma vez que tal processo apareceu como uma unidade de análise crucial para a compreensão do desenvolvimento humano. A inserção ecológica surgiu como alternativa metodológica para acessar os contextos de desenvolvimento, de forma que qualquer espaço em que se identifique o estabelecimento de processos proximais (ambiente doméstico, laboral, comunitário, escolar da pessoa, entre outros) torna-se propício à realização de pesquisas nesta perspectiva.

A criação do microssistema Juventude em Cena propiciou a reflexão sobre o que é estar ecologicamente inserido. Ao contrário das intervenções tradicionais da inserção ecológica, este programa criou um novo contexto para todos (Aquino-Morais, 2008; Cecconello, 2003; Morais, 2009). Nesse sentido, o estabelecimento dos processos proximais com outros sistemas ecológicos surgiu, ao longo das atividades estruturadas, mas principalmente, no decorrer dos momentos livres, como, por exemplo, horário de almoço e momentos culturais.

\section{Considerações finais}

Na perspectiva da ABDH, a pesquisa sobre o desenvolvimento humano preconiza a inclusão de experiências que visem à melhoria e à reestruturação dos sistemas ecológicos 
dominantes, de modo que as ideologias e regras institucionais preestabelecidas sejam reformuladas por meio da redefinição de objetivos, papéis e atividades que proporcionem a ligação a novos sistemas (Bronfenbrenner, 1977). Dessa maneira, o novo microssistema vem responder a essa demanda da ABDH. A construção de um novo microssistema caracterizou um espaço de transição ecológica, na medida em que relações foram estabelecidas, possibilitando o acesso de todos a conteúdos e experiências diversos. Por meio do Juventude em Cena, diferentes recursos - noções acerca dos direitos, da possibilidade de elaborar um projeto de vida e de buscar melhores condições de vida - foram disponibilizados, revelando a importância da ampliação dos multiambientes em que pessoas em desenvolvimento estavam inseridas. Quanto maiores as possibilidades de relações estabelecidas e a inserção em diferentes microssistemas, maiores as oportunidades de obtenção de recursos que favorecem o desenvolvimento humano. Considera-se que a ideia de dar continuidade ao programa de capacitação, a partir de novas edições, revela uma maneira de procurar assegurar o impacto dessa obtenção de recursos para o desenvolvimento humano.

A mudança de comportamentos e a aquisição de papéis vivenciados no novo microssistema proporcionaram transformações, as quais puderam ser transpostas aos demais sistemas - família, escola e comunidade. A ação protagonista idealizada pelo programa - que os adolescentes, cientes de seus direitos, pudessem buscar a garantia de tais direitos e melhores condições de vida - foi desenvolvida ao longo da intervenção. Naturalmente, foi observado como foi ampliada para outros contextos, na medida em que os adolescentes tornaram-se multiplicadores nas suas comunidades. Esse processo pôde ser acompanhado por meio da continuidade do programa e pela constatação de que alguns passaram a engajar-se em outras ações de participação social nos seus municípios de origem.

As dificuldades encontradas, ao longo do programa, permitiram refletir sobre a necessidade de estreitar parcerias com os profissionais e a rede de atenção infanto-juvenil dos municípios, buscando minimizar as barreiras que cada sistema apresenta. Assim, foram reunidos recursos de cada sistema, a fim de concretizar ações para o favorecimento do desenvolvimento das pessoas. A experiência vivenciada pela equipe e os resultados alcançados - maior engajamento nas atividades e interesse em ser protagonista, indo em busca dos seus direitos - enfatizam o impacto que intervenções deste tipo podem proporcionar.

A disponibilidade para o estabelecimento de relações recíprocas e a motivação pessoal da equipe foram um fator fundamental para a construção dos processos proximais e do novo microssistema. E, consequentemente a essas relações estabelecidas, mudanças foram alcançadas ao longo da intervenção, na medida em que os adolescentes se sentiam olhados, escutados, reconhecidos e valorizados. Conforme postulado por Bronfenbrenner (2005), foi possível observar, durante a execução do programa, que os processos proximais estão na base do desenvolvimento humano e que, por meio deles, resultados são alcançados.

Considera-se que a busca por uma construção de um pensamento político e crítico acerca dos direitos da criança e do adolescente e a proposta de ação protagonista dos adolescentes no meio em que eles vivem, a fim de gerar mudanças sociais, tiveram êxito ao longo do projeto. Estas transformações estão em consonância com o cerne ideológico da ABDH, a saber, a aplicação dos estudos em políticas públicas e sociais que possam fazer diferença na qualidade de vida das pessoas envolvidas (Bronfenbrenner, 2005).

Dos Quatro Pilares da Educação para o Desenvolvimento Humano (Hassenpflug, 2004), os que mais se salientaram foram o conhecer e o conviver, uma vez que os eixos temáticos visavam a instrumentalizar os adolescentes acerca de seus direitos, de valores de solidariedade e de cidadania. Entretanto, os pilares aprender a fazer e aprender a ser estavam claros no processo, configurando a articulação entre os restantes.

O programa Juventude em Cena proporcionou, por meio dos conteúdos trabalhados, das ações realizadas e das inter-relações estabelecidas, o desenvolvimento de todos em diversas dimensões humanas - cognitiva, social e pessoal. O trabalho executado promoveu o protagonismo juvenil, ao investir nas capacidades individuais dos adolescentes, auxiliando-os a percebê-las e se apropriarem delas. Nesse sentido, o programa fomentou o interesse dos participantes em buscar os seus direitos e incitou a possibilidade de mudanças nas comunidades de origem e em suas trajetórias de vida.

Este relato demonstra o importante papel da universidade que, além da formação de profissionais e a produção de conhecimento científico, cumpre sua função social por meio de ações de extensão capazes de contribuir para o desenvolvimento da comunidade. Ademais, como cientistas sociais e humanos, os profissionais da psicologia têm o compromisso de se aproximar da realidade das pessoas com as quais trabalham - em intervenções e pesquisas -, buscando compreender as características dos diversos sistemas. Desse modo, é construído um conhecimento que realmente oportunize uma vida mais digna. Programas que priorizem a participação juvenil, permitindo que os adolescentes expressem suas necessidades, poderão ser preventivos em relação a vários fatores de risco para essa parcela da população, suas famílias e a sociedade em geral. A universidade é um contexto propício para que iniciativas desse tipo sejam realizadas. Portanto, são necessários estudos futuros com análises mais aprofundadas e que demonstrem as mudanças, a partir da intervenção.

\section{Referências}

Aquino-Morais, C. (2008). Saúde, doença mental e serviços de saúde na visão de adolescentes e seus cuidadores. Dissertação de mestrado não-publicada, Universidade Federal do Rio Grande do Sul, Porto Alegre. 
Bronfenbrenner, U. (1977). Toward an experimental ecology of human development. American Psychologist, 32, 513-531.

Bronfenbrenner, U. (1988). Interacting systems in human development. Research paradigms: Present and future. In N. Bolger, A. Caspi, G. Downey, \& M. Moorehouse (Eds.), Persons in context: Development process (pp. 2549). New York: Cambridge Press.

Bronfenbrenner, U. (1993). The ecology of cognitive development: Research models and fugitive findings. In R. Worriak \& K. Fischer (Eds.), Development in context: Acting and thinking in specific environments (pp. 3-44). Hillsdale, NJ: Erlbawn.

Bronfenbrenner, U. (1995). Developmental ecology: Through space and time. In P. Moen, G. H. Elder, \& K. Luscher (Eds.), Examining lives in context: Perspectives on the ecology of human development (pp. 619-647). Washington, DC: APA.

Bronfenbrenner, U. (1996). A ecologia do desenvolvimento humano: Experimentos naturais e planejados (M. A. Veríssimo, Trad.) Porto Alegre: Artes Médicas. (Original publicado em 1979)

Bronfenbrenner, U. (2005). The bioecological theory of human development. In U. Bronfenbrenner (Ed.), Making human beings humans (pp. 3-15). Thousand Oaks, CA: Sage.

Bronfenbrenner, U., \& Evans, G.W. (2000). Development science in the 21st century: Emerging questions, theoretical models, research designs and empirical findings. Social Development, 9, 115-125.

Bronfenbrenner, U., \& Morris. P.A. (1998). The ecology of developmental processes. In W. Damon \& R. M. Lerner (Eds.), Handbook of child psychology: Theoretical models of human development (Vol. 1, pp. 993-1027). New York: John Wiley \& Sons.

Cecconello, A. M. (2003). Resiliência e vulnerabilidade em famílias em situação de risco. Tese de doutorado nãopublicada, Universidade Federal do Rio Grande do Sul, Porto Alegre.

Cecconello, A. M. \& Koller, S. H. (2003). Inserção ecológica na comunidade: Uma proposta metodológica para o estudo de famílias em situação de risco. Psicologia: Reflexão e Crítica, 16, 515-524.

Delors, J. (Org.). (1998). Educação: Um tesouro a descobrir. Relatório para a Unesco da Comissão Internacional sobre Educação para o século XXI. São Paulo: Cortez/Brasília/ MEC/UNESCO.

Eschiletti Prati, L., Paula Couto, M. C. P., Moura, A. S., Poletto, M., \& Koller, S. H. (2008). Revisando a inserção ecológica: Uma proposta de sistematização. Psicologia: Reflexão e Crítica, 21, 160-169.

Hassenpflug, W. N. (2004). Educação pelo esporte: Educação para o desenvolvimento humano pelo esporte. São Paulo: Saraiva/Instituto Ayrton Senna.
Lei No. 8.069, de 13 de julho de 1990. (1990, 13 de julho). Estatuto da Criança e do Adolescente. Diário Oficial da União, seção 1.

Morais, N. A. (2009). Trajetórias de vida de crianças e adolescentes em situação de vulnerabilidade social: Entre o risco e a proteção. Tese de doutorado nãopublicada, Universidade Federal do Rio Grande do Sul, Porto Alegre.

Organização das Nações Unidas. (1989). Convenção sobre os direitos da Criança. Recuperado em 09 outubro 2009, de http://www.unicef.org.brazil/dir_cri.htm

Vasconcelos, Q. A., Yunes, M. A. M., \& Garcia, N. M. (2009). Um estudo ecológico sobre as interações da família com o abrigo. Paidéia (Ribeirão Preto), 19, 221-229.

Verhellen, E. (2000). Convention on the rights of the child. Leuven, Belgium: Garant.

Ana Paula Lazzaretti de Souza é doutoranda do Programa de Pós-graduação em Psicologia do Instituto de Psicologia da Universidade Federal do Rio Grande do Sul, bolsista CAPES.

Luciana Dutra-Thomé é doutoranda do Programa de Pósgraduação em Psicologia do Instituto de Psicologia da Universidade Federal do Rio Grande do Sul, bolsista CAPES.

Eva Diniz Bensaja Dei Schiró é doutoranda do Programa de Pós-graduação em Psicologia do Instituto de Psicologia da Universidade Federal do Rio Grande do Sul, bolsista CNPq. Camila de Aquino Morais é doutoranda do Programa de Pósgraduação em Psicologia Clínica e Cultura do Instituto de Psicologia da Universidade de Brasília, bolsista CNPq. Silvia Helena Koller é Professora Associada do Programa de Pós-graduação em Psicologia do Instituto de Psicologia da Universidade Federal do Rio Grande do Sul.

Recebido: 04/09/2009

$1^{a}$ revisão: 08/02/2010

$2^{a}$ revisão: $28 / 10 / 2010$

Aceite final: 26/11/2010 